\title{
Beyond linear conciliation
}

\author{
Ko-Hung Kuan ${ }^{1}$
}

Received: 7 September 2019 / Accepted: 24 July 2020 / Published online: 6 August 2020 (c) The Author(s) 2020

\begin{abstract}
Formal epistemologists criticise the Conciliatory View of peer disagreement for being non-commutative with conditionalisation, path dependent and does not preserve the independence between propositions. Failing to commute with conditionalisation, one may switch the order between conciliating and conditionalising and obtain different outcomes. Failing to be path independent, the outcome of conciliation varies with the order of the acquisition of new testimonies. Failing to preserve the independence between propositions, one may suffer from a sure-loss and hence be deemed irrational. The three formal deficiencies urge people to abandon the Conciliatory View. This paper aims to show that one may save the Conciliatory View by conciliating with nonlinear functions. Research in the study of opinion pooling shows that the three deficiencies are not problems of the Conciliatory View, but problems of linear averaging. Hence, one can get rid of these formal deficiencies by making conciliation with nonlinear averaging functions. After showing how the three deficiencies can be avoided, I will explore the features of nonlinear averaging functions and argue that they have properties that correctly capture people's intuition concerning disagreement. The conclusion, therefore, is to suggest epistemologists develop a more fine-grained taxonomy for cases of disagreement. With a deliberate categorisation of different kinds of disagreement, epistemologists can pick the proper averaging rule to apply in each specific case, and get rid of possible formal deficiencies.
\end{abstract}

Keywords Formal epistemology $\cdot$ Social epistemology $\cdot$ Opinion pooling $\cdot$ Peer disagreement $\cdot$ Nonlinear averaging

Ko-Hung Kuan

k.kuan@1se.ac.uk

1 Department of Philosophy, Logic and Scientific Method, London School of Economics, Houghton Street, London WC2A 2AE, UK 


\section{Introduction}

The Conciliatory View of peer disagreement holds that when one disagrees with their epistemic peers, one should compromise with their peers by revising their credence in the proposition at issue (Christensen 2007; Elga 2007; Feldman 2006). ${ }^{1}$ Despite its intuitive plausibility, many epistemologists find this view untenable. Some claim that conciliating is a self-abasing act (Pettit 2006; van Inwagen 1996), while others argue that it is not truth-conducive (Kelly 2010). Among the arguments against the Conciliatory View, the ones that focus on its formal deficiencies deserve special attention. It has been pointed out that there are three deficiencies in the Conciliatory View. First, it does not commute with the Bayesian rule of conditionalisation since the outcome of conciliation is partially determined by whether one updates before making conciliation (Fitelson and Jehle 2009; Wilson 2010). Second, the Conciliatory View is path dependent (Gardiner 2014). In a case where one makes multiple conciliations with their peers at different times, the final result is determined by the temporal order in which one makes the conciliations with each peer. Third, the Conciliatory View does not preserve one's judgement concerning the relevance between propositions (Elkin and Wheeler 2018). That is, one's judgement that two propositions are irrelevant may not be well preserved after they conciliates with others. Since all three features bring about some unacceptable consequences, the Conciliatory View seems seriously flawed.

The criticisms concerning the formal deficiencies of the Conciliatory View, however, are misguided. Studies in opinion pooling have shown that it is linear averaging, the function that is generally adopted to make conciliation, that has the three formal deficiencies. Since linear averaging is by no means the only legitimate way to make conciliation, one can save the Conciliatory View by adopting nonlinear average functions. To show this, I will first introduce the Conciliatory View and its most prominent form, the Equal Weight view. After reformulating the two views in a formal framework, I will demonstrate the three formal deficiencies of the Conciliatory View and explain how can they be solved by making conciliation with geometric and multiplicative average functions. To further justify the approach of nonlinear conciliation, I will point out that some features of the nonlinear average functions better reflect our intuitions about disagreement. As a result, some misconceptions in the study of peer disagreement may be clarified. The conclusion, hence, is that we should embrace a pluralistic view concerning conciliation and give up the idea that there is a single conciliating rule which can be applied in every case of peer disagreement. Conciliationists should develop a taxonomy of different kinds of disagreement and find out the proper average function to apply for each kind.

\section{Disagreement between peers}

Consider the following scenario: Albert, a brilliant historian who specialises in the Victorian era, wants to solve the mystery of Jack the Ripper. Having spent years

\footnotetext{
${ }_{1}^{1}$ The formulation here involves the notion of credence. I will provide a reason for formulating this way in Sect. 3 .
} 
reviewing all the evidence relevant to the Whitechapel murders, he becomes very confident that it was the Polish barber Aaron Kosminski who committed the atrocity. However, his colleague Bridget firmly believes the opposite. Like Albert, Bridget is also an expert in Victorian Britain who has reviewed all the evidence related to the murders. Unlike Albert, Bridget considers it extremely unlikely that Kosminski is Jack the Ripper. Knowing that Bridget, as a historian, is as good as himself, how should Albert respond to their disagreement concerning this controversy?

Albert and Bridget's case is a typical example of peer disagreement. Two symmetric assumptions need to be highlighted for one to see why these kinds of circumstances constitute a real problem for epistemologists. First, since it is assumed that Bridget is Albert's epistemic peer, they are symmetric with their reliability concerning this issue. We may unpack this assumption a bit further by assuming that Albert and Bridget were both educated in prestigious universities, trained in similar ways and had equally outstanding track records. Given these conditions, they are equally likely to have the correct credence concerning the historical fact in question with the same body of evidence. Precisely because of such peerhood, any reason which allows Albert to cast doubt on Bridget's credences concerning specific historical facts should also allow Bridget to cast doubt on Albert's credences. Hence, Albert cannot dismiss Bridget's disagreement but must take it seriously. Second, they are symmetric with the evidence they possess respectively. The evidence Albert has is to a great extent, if not exactly, identical to the evidence Bridget has. ${ }^{2}$ Because of the parity of evidence between them, one cannot expect either of them to change their mind after reviewing the evidence available to themselves. It is the two symmetries that make peer disagreement a thorny problem for social epistemologists.

There are many variants of the standard peer disagreement case that can be generated by revising the two symmetric assumptions. Regarding the symmetry of reliability, one may specify the reliability of the interlocutors involved. When all the interlocutors are highly reliable, we have the case of expert disagreement. On the contrary, when all the interlocutors are unreliable, we have the case of layperson disagreement. Regarding the symmetry of evidence, one may assume different degrees of information they have concerning the evidence their peers possess. In some cases, all the interlocutors are fully aware that others have the same body of evidence as they do. Each of them not only knows that the other interlocutors do possess some evidence but also knows the content of the evidence. In a slightly different case, the interlocutors do not share the full content of the evidence. What they know is that all the interlocutors possess evidence of the same strength. ${ }^{3}$ If we relax the notion of peerhood further, we may derive cases in which the interlocutors know that the other interlocutors have some pieces of evidence, but have no information concerning the strength of the evidence others possess. The most radical case would be one where each interlocutor has no idea whether their peers have any piece of evidence. Due to the highly varied nature of all these different kinds of cases, they should be treated in different ways.

\footnotetext{
${ }^{2}$ Some epistemologists consider cases in which the individuals have pieces of evidence that are only accessible to themselves. Here it is assumed that this kind of evidence does not play a crucial role in their judgement.

3 This definition of peer disagreement can be found in Matheson (2014).
} 
One may doubt whether these variants still count as peer disagreement, especially cases of the latter kind in which the symmetry of evidence is weakened. Indeed, without assuming the symmetry of evidence, the problem of peer disagreement might be thought to be overly easy and loses its philosophical significance. However, as King (2012) points out, a perfect case of peer disagreement, namely one which satisfies both symmetric assumptions, is rather rare. The ultimate goal of the study of peer disagreement should not be finding a solution that is only applicable to the hardest cases. If we take it to be the final goal, the study of peer disagreement may be somewhat trivial as the result is extremely limited. What we should aim for, instead, is finding out a solution that applies to a wider set of cases. ${ }^{4}$ Following this line of thought, then, cases without the symmetry of evidence, though deviant from the standard cases of peer disagreement, are still worthy discussed.

\section{The Conciliatory View and the Equal Weight View}

One of the most widely accepted solutions to peer disagreement is the Conciliatory View: Whenever a disagreement occurs among a group of epistemic peers, each one involved should make a compromise with the others. The motivation is reasonably straightforward: Since no one is epistemically impeccable, it is always possible for an individual to have incorrect credence in a proposition. Thus, when one disagrees with their epistemic peers, one should realise that they might have made a mistake and revise their credence in the proposition in doubt. We can perhaps see the plausibility of this view from another perspective. In the face of peer disagreement, a person who refuses to change their credence can be criticised for ignoring their own fallibility. To be epistemically modest, one should choose to conciliate when involved in a disagreement.

A question immediately follows: How, in practice, should one make a conciliation? Conciliation can be made in many different ways. One can conciliate either by giving up their credence entirely and accept whatever their peers say, or by making a minimal revision of their original credence concerning the proposition in question. Although the two ways lead to remarkably different outcomes, they both count as conciliating. If the Conciliatory View suggests a variety of ways of dealing with disagreement, it would be overly general and hence lack significance. Thus, conciliationists cannot merely claim that conciliation is the proper solution to peer disagreement, but have to provide precise instructions concerning how people should revise their credence in the face of disagreement.

One way to establish a more elaborate formulation of the Conciliatory View is to reconsider the core assumption of peer disagreement. Recall that all the individuals involved in disagreement are assumed as equally reliable. All their credences concerning the proposition in dispute, therefore, are equally likely to be correct and should be treated in the same way. Thus, the most promising form of the Conciliatory View is to assign equal weight to all the disagreeing peers' credences and take the average as

\footnotetext{
4 Matheson (2014) also holds the view that the purpose of studying peer disagreement is to find a solution applicable to other cases.
} 
the outcome of conciliation. Call this the Equal Weight View (henceforth the EWV). ${ }^{5}$ Take the Jack the Ripper controversy for example. In the given scenario, Albert is nearly sure that Aaron Kosminski is the one who committed the Whitechapel murders, while Bridget is almost certain that Kosminski is not. If Albert adopts the EWV and assigns equal weight to both his credence and Bridget's, he would come to have moderate credence in the proposition under dispute. In the following sections, I will take the EWV as a view representing other Conciliatory Views since they share all the important formal properties which we are going to discuss.

The core idea of the EWV, as stated, is to respect the fact that all the individuals involved in a genuine peer disagreement are equally reliable. Hence, everyone's opinion should be given equal weight. From this core claim, one may infer that the EWV should be formulated within a Bayesian framework which represents an individual's doxastic state as credence. ${ }^{6}$ To see this, consider a case where three individuals $A, B$ and $C$ disagree over the truth of a proposition. Both $A$ and $B$ believe that the proposition is true, while $C$ disbelieves. Suppose that they all adopt the EWV and intend to revise their credence in the proposition, what would the outcome be? There is no answer if we adopt the traditional tripartite conception of belief which says that one either believes, disbelieves or suspends judgement concerning a proposition. The individuals should not jointly believe or disbelieve the proposition since there is no consensus among them. The remaining option, namely suspend judgement, is also incorrect. A joint suspension of judgement concerning the issue implies that $C$ 's disbelief outweighs $A$ and $B$ 's beliefs, which leads to a violation of the EWV. If instead of taking this overly coarse-grained framework, we choose to represent their doxastic states in terms of credences, this problem can be solved easily. In brief, since credences can be properly split and represent all the possible outcomes of conciliation, the EWV should be formulated formally. Following the same line of reasoning, any non-trivial form of the Conciliatory View is also fundamentally formal. ${ }^{7}$

Since the EWV is essentially formal, we need to formulate it within a formal framework. The first item required is the set of all the propositions that can be subject to disagreement. Take a non-empty finite set of possible worlds $\Omega$ as primitive, a single proposition can be defined as a subset of $\Omega$, which is the set of all the worlds where the proposition is true. The set of all propositions, following this definition, should be defined as $2^{\Omega}$, namely the power set of $\Omega$. An individual's credence over each proposition can hence be defined as a function. Let $\operatorname{Pr}_{i}(\cdot)$ represent the credence function of individual $i$ which assigns a value in the interval $[0,1]$ to every possible world $\omega$ in the set $\Omega$, where the sum total of values across the worlds in $\Omega$ is 1 . As a direct result, every credence function also assigns a value to every proposition in $2^{\Omega}$, namely the sum of its values for the constituent worlds. Every credence function is formally a probability function.

\footnotetext{
5 For a detailed introduction to the EWV, see Feldman (2006), Elga (2007) and Christensen (2007).

6 The EWV can also be formulated in terms of imprecise probability. See Elkin and Wheeler (2018).

7 Kelly (2010, p. 187) has another argument supporting the claim that the EWV should be presented within a formal framework.
} 
The most widely accepted version of the EWV takes the linear average of the disagreeing individuals' credences as the outcome of conciliation, which can be formulated as the following:

Definition 1 The Linear Equal Weight View

Given a case in which $n$ individuals $1, \ldots, n$ disagree over the proposition $P \in 2^{\Omega}$, the outcome of conciliation should be

$$
\sum_{i=1}^{n} \frac{1}{n} \operatorname{Pr}_{i}(P) .
$$

That is, one may divide the sum of individual credences with the number of individuals involved and take the outcome as the result of conciliation.

An example may illustrate how the Linear EWV works. Suppose that, in the Jack the Ripper case, Albert's credence in Kosminski being Jack the Ripper $(P)$ is 0.9 while Bridget's is 0.1 . Taking their credence functions respectively as $\operatorname{Pr}_{1}(\cdot)$ and $\operatorname{Pr}_{2}(\cdot)$, the result of assigning equal weight to both their credences in $P$ is:

$$
\frac{1}{2} \operatorname{Pr}_{1}(P)+\frac{1}{2} \operatorname{Pr}_{2}(P)=\frac{1}{2} \cdot 0.1+\frac{1}{2} \cdot 0.9=0.5
$$

According to the Linear EWV, they should have 0.5 credence in Kosminski being the real murderer. This result correctly captures our intuition that they should both have moderate credence over $P$ after conciliating with each other.

\section{Three formal deficiencies of the Linear Conciliatory View}

\subsection{Non-commutativity with conditionalisation}

Although the Linear EWV seems to be an ideal rule for making conciliation, it has three major formal deficiencies. First of all, it fails to commute with the Bayesian rule of conditionalisation. As one of the defining features of Bayesianism, conditionalisation suggests any individual who acquires a piece of evidence $E$ update their credence by conditioning their credence in any proposition on $E$. Apart from being a handy and plausible rule for updating credence, conditionalisation is also the Bayesian norm for the diachronic coherence of one's credences. If one does not update their credence with the rule of conditionalisation upon receiving new evidence, one takes the risk of having diachronically incoherent credences over a set of propositions. ${ }^{8}$

It has been pointed out by many epistemologists that the Linear EWV fails to commute with conditionalisation as switching the order between conciliating and updating leads to different outcomes (Fitelson and Jehle 2009; Wilson 2010). To

\footnotetext{
8 Although the rule of conditionalisation is a core Bayesian norm, it is not undoubtedly true. Some philosophers argue that one may reject conditionalisation yet still be rational (Bacchus et al. 1990; Hild 1998; Arntzenius 2003). For the projects aiming at vindicating conditionalisation, see Greaves and Wallace (2005), Briggs and Pettigrew (2020) and Pettigrew (2019).
} 
Table 1 Albert and Bridget's credences over $P$ and $E$

\begin{tabular}{lllll}
\hline$P$ & $E$ & $P r_{1}(\cdot)$ & $P r_{2}(\cdot)$ & $P r_{1+2}(\cdot)$ \\
\hline $\mathrm{T}$ & $\mathrm{T}$ & 0.285 & 0.05 & 0.1675 \\
$\mathrm{~T}$ & $\mathrm{~F}$ & 0.615 & 0.05 & 0.3325 \\
$\mathrm{~F}$ & $\mathrm{~T}$ & 0.015 & 0.15 & 0.0825 \\
$\mathrm{~F}$ & $\mathrm{~F}$ & 0.085 & 0.75 & 0.4175 \\
\hline
\end{tabular}

illustrate, consider the Jack the Ripper example. Let $\operatorname{Pr}_{1}(\cdot)$ stand for Albert's credence function, $\operatorname{Pr}_{2}(\cdot)$ for Bridget's and $\operatorname{Pr}_{1+2}(\cdot)$ for their joint credence function obtained by conciliating with the Linear EWV. Assume that they have the following credences over the proposition $P$ and a piece of evidence $E$ :

The first row stands for the possible world in which $P$ and $E$ are both true. The value given by $\operatorname{Pr}_{1}(\cdot)$, hence, is Albert's credence in $P \& E$. Given this credence distribution, if Albert and Bridget decide to first make a conciliation concerning their credences in $P$ and $E$, their joint credence in $P \& E$ and $E$ would respectively be the following:

$$
\begin{aligned}
& \operatorname{Pr}_{1+2}(P \& E)=\frac{1}{2} \operatorname{Pr}_{1}(P \& E)+\frac{1}{2} \operatorname{Pr}_{2}(P \& E)=\frac{1}{2}(0.285+0.05)=0.1675, \\
& \operatorname{Pr}_{1+2}(E)=\frac{1}{2} \operatorname{Pr}_{1}(E)+\frac{1}{2} \operatorname{Pr}_{2}(E)=\frac{1}{2}((0.285+0.015)+(0.05+0.15))=0.25 .
\end{aligned}
$$

By applying the rule of conditionalisation, we may derive their joint credence of $P$ conditional on $E$ :

$\operatorname{Pr}_{1+2}(P \mid E)=\frac{\operatorname{Pr}_{1+2}(P \& E)}{\operatorname{Pr}_{1+2}(E)}=\frac{\frac{1}{2}\left(\operatorname{Pr}_{1}(P \& E)+\operatorname{Pr}_{2}(P \& E)\right)}{\frac{1}{2}\left(\operatorname{Pr}_{1}(E)+\operatorname{Pr}_{2}(E)\right)}=\frac{0.1675}{0.25}=0.67$.

On the other hand, if Albert and Bridget choose to first update their credences on the evidence $E$ respectively, they would have the following credences in $P$ conditional on $E$ :

$$
\begin{aligned}
& \operatorname{Pr}_{1}(P \mid E)=\frac{P r_{1}(P \& E)}{P r_{1}(E)}=\frac{0.285}{0.3}=0.95 \\
& \operatorname{Pr}_{2}(P \mid E)=\frac{P r_{2}(P \& E)}{P r_{2}(E)}=\frac{0.05}{0.2}=0.25 .
\end{aligned}
$$

If they, after updating with $E$ respectively, make a conciliation, their joint credence would be the linear average of the two values:

$$
\left.\operatorname{Pr}_{1+2}(P \mid E)=\frac{1}{2} \operatorname{Pr}_{1}(P \mid E)+\frac{1}{2} \operatorname{Pr}_{2}(P \mid E)\right)=\frac{1}{2}(0.95+0.25)=0.6 .
$$

This case shows that if the individuals make conciliation with the Linear EWV, the order of conciliating and updating determines the outcome of their conciliation. 
What is wrong with the Linear EWV failing to commute with conditionalisation? First, the order of updating and conciliating is, in most cases, irrelevant to the disagreement itself. It is unacceptable to let an irrelevant factor determine the outcome of conciliation. Suppose that, in the Jack the Ripper case, Albert and Bridget decide to conciliate with each other and have moderate credence in Kosminski being the murderer. After the conciliation, they find a ledger containing the names of suspects that they have never seen. Both Albert and Bridget update with this new piece of evidence and come to have a new credence in Kosminski being the murderer. In a different case, they find the ledger and each update their credence before they conciliate. Since conciliation does not commute with conditionalisation, the result of conciliation in the second case may differ from the result in the first case. This is rather problematic since the time they receive the ledger is irrelevant to whether Kosminski is Jack the Ripper. If the purpose of conciliating is to make disagreeing peers come to have credences that are as close to the truth as possible, we should not take an irrelevant factor into account. Second, if the outcome of conciliation is sensitive to the time of updating, the individuals involved in disagreement would be vulnerable to manipulation. Suppose that, in the Jack the Ripper case, another historian Claire possesses a new piece of evidence $E^{\prime}$ which is unknown to both Albert and Bridget. Further suppose that Claire intends the outcome of Albert and Bridget's conciliation to be as close to 1 as possible. Knowing that Albert and Bridget are about to conciliate, Claire would choose to conceal $E^{\prime}$ until the conciliation is made. By revealing $E^{\prime}$ to Albert and Bridget after they make a conciliation, she can make their joint credence closer to 1 , which is the result she intends. This feature is again undesirable, as an ideal rule should leave no space for manipulation. In other words, conciliating with the Linear EWV puts people under the risk of being manipulated. The Linear EWV, therefore, is a problematic way of resolving peer disagreement.

\subsection{Path dependence}

Apart from failing to commute with conditionalisation, the Linear EWV also fails to be path independent (Gardiner 2014). That is, if one makes multiple conciliations according to the Linear EWV, the outcome would be sensitive to the order of conciliation. Imagine a revised Jack the Ripper case in which another historian Claire has credence 0.7 in the proposition $P$ that Kosminski is Jack the Ripper. Suppose, as before, that Albert's credence in $P$ is 0.9 while Bridget's is 0.1 . If Albert first makes a conciliation with Claire and subsequently with Bridget, his credence in $P$, according to the Linear EWV, would be 0.45 . Let Claire's credence function be $\operatorname{Pr}_{3}(\cdot)$, the process can be formulated as the following:

$$
\begin{aligned}
& \operatorname{Pr}_{1+3}(P)=\frac{0.9+0.7}{2}=0.8 \\
& \operatorname{Pr}_{1+3+2}(P)=\frac{0.8+0.1}{2}=0.45
\end{aligned}
$$

After Albert conciliates with Claire, they obtain the joint credence function $P_{1+3}(\cdot)$ which assigns $P$ with credence 0.8 . When they subsequently make a conciliation with 
Bridget, the resulting credence function is $\operatorname{Pr}_{1+3+2}(\cdot)$ which assigns 0.45 to $P$. In a slightly different story, Albert first conciliates with Bridget and subsequently with Claire. His credence in $P$, given the Linear EWV, would be 0.6 . That is,

$$
\begin{aligned}
& \operatorname{Pr}_{1+2}(P)=\frac{0.9+0.1}{2}=0.5 \\
& \operatorname{Pr}_{1+2+3}(P)=\frac{0.5+0.7}{2}=0.6
\end{aligned}
$$

Given this example, we may see that the order Albert conciliates with his peers determines the outcome of conciliation.

Path dependence is undesirable for two reasons. First, the order one makes conciliation with their peers is, in most cases, irrelevant to the proposition in dispute and should not affect the outcome of conciliation in any way. Consider the Jack the Ripper case again. Whether Kosminski committed the Whitechapel murders is a historical event that has already obtained. The temporal order Albert makes conciliation with his peers has nothing to do with the real identity of Jack the Ripper. Hence, it would be absurd to let the order Albert conciliates be a factor determining the outcome of conciliation. Moreover, the result shows that the Linear EWV is diachronically incoherent. The core claim of the EWV is that every individual's credence should be treated equally. If one adopts the Linear EWV and makes conciliation with their peers one at a time, the testimonies that are received at some early stage would be weighted less than the testimonies that come later, as earlier testimonies have been mixed up with new testimonies for more times. In other words, the importance of a single testimony would be gradually diluted as new pieces of testimonial evidence emerge. As this result violates the core idea of the EWV that one should weight all their peers' credences equally, the Linear EWV runs the risk of being self-refuting.

One might attempt to defend the Linear EWV by arguing that people can assign weights in a more sophisticated way. Instead of reassigning equal weight in every single conciliation, an individual should keep track of all the conciliations they have made and assign the correct weight to new peers. In the given example, after Albert conciliates with Bridget, he should be aware of the fact that his credence has already been mixed up with Bridget's prior credence. When Albert subsequently meets Claire and makes another conciliation, he should know that the correct weight to assign to Claire's credence is one-third rather than a half. In short, if Albert is smart enough, he should know the correct weight to assign to his peers' credence. The problem of path dependence only occurs to stubborn individuals.

Although the solution is rather convincing, it is difficult to implement in most cases of peer disagreement. In a simple case which involves only a small number of individuals, it is relatively easy for one to remember the details of all the conciliations they have made. However, in a slightly complicated case, it would be overly demanding to ask an individual to memorise all the details of every conciliation that has taken place. Suppose that Albert, in the searching of the true identity of Jack the Ripper, consults twenty peers at different times. It would be extremely tough for him to remember every peer's prior credence and assign the correct weight to each of them. Hence, it is 
pragmatically impossible for one to always conciliate with their peers this way. ${ }^{9}$ For this reason, path dependence remains a defect of the Linear EWV.

Another way of saving the Linear EWV is to deny that one would ever need to make multiple conciliations with their peers. In the given scenario, after Albert conciliates with Bridget, it can be said that Claire is no longer his peer since, after the conciliating with a peer, Albert's credence has become more likely to be correct. Hence, Albert is no longer Claire's peer and does not have to assign equal weight to her credence. The problem of path dependence, therefore, would not occur in the first place. However, this solution is based on a volatile notion of peerhood. In any ordinary case of peer disagreement, one does not become superior immediately after they conciliate with a single peer. Imagine a case where a panel of scientists aims to make a joint decision concerning government policies. Two among them have a private conversation and, right after they make a conciliation, announce that their credence over the proposition in question is the correct one since they have become superior to all other scientists. If one agrees that this case is absurd, they would have to admit that a proper notion of peerhood should prevent this kind of things from happening. For this reason, we may conclude that path dependence remains a shortcoming of the EWV.

\subsection{The problem of independence preservation}

Another crucial problem of the Linear Conciliatory View is that it fails to preserve their judgement of independence (Bradley et al. 2014; Elkin and Wheeler 2018). Let us consider a concrete example provided by Elkin and Wheeler (2018): Albert, based on his evidence, does not find it likely that it will rain in Kyoto tomorrow $(K)$ and has 0.2 credence in the proposition. On the other hand, with some evidence, he thinks that there may be an unpublished volume of The Lord of the Rings $(R)$ and has 0.4 credence in its existence. Based on the same background knowledge and evidence, Bridget has 0.6 credence in the former proposition and 0.55 in the latter. Further suppose that Albert judges $K$ and $R$ as mutually independent. His credence in the conjunction of $K$ and $R$, thus, is equal to the product of his credence in $K$ and his credence in $R$, namely 0.16 . Bridget also judges $K$ and $R$ as mutually independent and has 0.33 credence in the conjunction of $K$ and $R$. When they conciliate with each other according to the Linear EWV, they come to accept a new credence function $\operatorname{Pr}_{1+2}(\cdot)$ which does not assign equal value to the conjunction of $K$ and $R$ and the product of their respective joint credence in $K$ and $R$. That is, $K$ and $R$ are not independent according to their joint credence function $\operatorname{Pr}_{1+2}(\cdot)$. The distribution of their credences is presented in Table $2 .{ }^{10}$

Intuitively, this result is strange. As both Albert and Bridget judge $K$ and $R$ as mutually independent, the joint judgement that $K$ and $R$ are relevant, revealed by the joint credence function $\operatorname{Pr}_{1+2}(\cdot)$, comes from nowhere. A more serious problem is that failing to preserve ones' judgement of independence may make them irrational. Suppose that Albert and Bridget do make conciliation according to the Linear EWV and adopt $\operatorname{Pr}_{1+2}(\cdot)$ as their new credence function. A witty gambler can sell them two

\footnotetext{
9 Gardiner (2014) provides a thorough review of the possible solutions to this problem.

10 Thanks to an anonymous referee for indicating that this problem should be discussed.
} 
Table 2 Albert $\left(\operatorname{Pr}_{1}(\cdot)\right)$ and Bridget's $\left(\operatorname{Pr}_{2}(\cdot)\right)$ credences in $K$ and $R$

\begin{tabular}{lllll}
\hline & $P r_{1}(\cdot \wedge \cdot)$ & $P r_{2}(\cdot \wedge \cdot)$ & $P r_{1+2}(\cdot \wedge \cdot)$ & $P r_{1+2}(\cdot) P r_{1+2}(\cdot)$ \\
\hline$K, R$ & 0.08 & 0.33 & 0.205 & 0.19 \\
$K, \neg R$ & 0.12 & 0.27 & 0.195 & 0.21 \\
$\neg K, R$ & 0.32 & 0.22 & 0.27 & 0.285 \\
$\neg K, \neg R$ & 0.48 & 0.18 & 0.33 & 0.315 \\
\hline
\end{tabular}

bets: The first bet costs them $\$ 20.5$ on the condition that they receive $\$ 100$ from the gambler if $K$ and $R$ are both true. The second bet costs them $\$ 33$ on the condition that the gambler pays them $\$ 100$ if $K$ and $R$ are both false. Since, according to the function $\operatorname{Pr}_{1+2}(\cdot \wedge \cdot)$, Albert and Bridget's joint credence in both $K$ and $R$ being true is 0.205 , the expected return of the first bet for them is 0. Similarly, since their joint credence in $K$ and $R$ both being false is 0.33 , the expected return of the second bet is also 0 . As both bets are fair for Albert and Bridget, they will accept both bets.

The gambler can go further and sell Albert and Bridget two other bets: One costs them $\$ 21$ and pays back $\$ 100$ if $K$ is true and $R$ is false. Another costs them $\$ 28.5$ and pays back $\$ 100$ if $K$ is false and $R$ is true. Since both Albert and Bridget judge $K$ and $R$ as independent, their credence in $K$ and $R$ can also be represented by the function $\operatorname{Pr}_{1+2}(\cdot) \operatorname{Pr}_{1+2}(\cdot)$. Given this credence function, the two new bets are fair. Hence, Albert and Bridget would also accept this proposal. With the four bets, it is guaranteed that Albert and Bridget are going to lose $\$ 3$ to the gambler. This case shows that adopting the Linear EWV may lead to a sure loss of money, which indicates that the individuals are irrational. The linear EWV, therefore, should be abandoned.

\section{Opinion pooling and peer disagreement}

One way of saving the Conciliatory View is to change the way we conciliate while retaining the core idea that one should make conciliation with their peers. Since it has been proved that there are nonlinear average functions that are free from the three formal problems, we may adopt the nonlinear average functions to make conciliation and thereby derive alternative Conciliatory Views that are free from the three formal deficiencies. $^{11}$

The study of probabilistic opinion pooling aims to answer one central question: Given a profile of credence functions across a set of individuals, what is the proper way of merging them into a single joint credence function which satisfies specific requirements? To correctly respond to this question, philosophers have examined a variety of possibilities and proposed different pooling functions. Since, as we have seen, the Conciliatory View is essentially formal, we may apply the results in the study of opinion pooling to save this view. In the following sections, I will show that nonlinear average functions are free from the three formal deficiencies of the Linear

\footnotetext{
11 This idea is first proposed by Martini et al. (2013). However, they did not fully explore the possible outcomes of adopting nonlinear functions to make conciliation.
} 
EWV. As the formal results derived can be generalised to other forms of conciliation, the Conciliatory View can be rescued.

It has been proven that the geometric averaging does commute with conditionalisation (Genest 1984). Hence, we may adopt geometric averaging to make conciliation and thereby derive a rule of conciliation that commutes with conditionalisation:

Definition 2 The Geometric Conciliatory View

Given a case in which $n$ individuals $1, \ldots, n$ disagree over the proposition $P$, the Geometric Conciliatory View suggests the individuals involved to have credence

$$
\sum_{\omega \in P} c \cdot \operatorname{Pr}_{1}(\omega)^{w_{1}} \cdots \operatorname{Pr}_{n}(\omega)^{w_{n}}
$$

in the proposition $P$, where the factors $w_{1}, \ldots, w_{n}$ are the weights assigned to each individual which sum up to 1 , and the constant $c$ is a normalisation factor which guarantees that the sum of joint credences across all worlds equals 1 :

$$
c=\frac{1}{\sum_{\omega^{\prime} \in \Omega} \operatorname{Pr}_{1}\left(\omega^{\prime}\right)^{w_{1}} \cdots \operatorname{Pr}_{n}\left(\omega^{\prime}\right)^{w_{n}}} .
$$

A technical point to be highlighted is that the credence functions here take a single possible world, rather than a proposition, as its input. ${ }^{12}$ The credence each individual has in a proposition can be derived by summing up the credence over each possible worlds included in the proposition. ${ }^{13}$

To show that the Geometric Conciliatory View does commute with conditionalisation, we need to reformulate the rule of conditionalisation. ${ }^{14}$ Given any piece of evidence $E$, the information it carries allows the individuals to derive a likelihood function $L$ which assigns either the value 1 or 0 to each possible world, representing the degree $E$ supports each world $\omega$. For example, if a world $\omega_{k}$ is in $E$, then $L\left(\omega_{k}\right)=1$. An individual $i$ can then update their credence function $P$ as

$$
\operatorname{Pr}_{i}^{L}(\omega)=\frac{\operatorname{Pr}_{i}(\omega) L(\omega)}{\sum_{\omega^{\prime} \in \Omega} \operatorname{Pr}_{i}\left(\omega^{\prime}\right) L\left(\omega^{\prime}\right)}
$$

which is equivalent to

$$
\operatorname{Pr}_{i}^{E}(\omega)=\frac{\operatorname{Pr}_{i}(\omega) \operatorname{Pr}_{i}(E \mid \omega)}{\sum_{\omega^{\prime} \in \Omega} \operatorname{Pr}_{i}\left(\omega^{\prime}\right) \operatorname{Pr}_{i}\left(E \mid \omega^{\prime}\right)}
$$

Since this formula is the rule of conditionalisation in a different form, updating with a likelihood function is equivalent to updating with conditionalisation.

\footnotetext{
12 This is in line with the definition that each credence function assigns a value to each possible world.

13 For a detailed explanation, see Dietrich and List (2016, p. 8).

14 The likelihood function, compared to Bayesian conditionalisation, is actually a more general update rule, as it can take any possible value, rather than the discrete values 1 and 0 . From this perspective, Bayesian conditionalisation is a limiting case of updating by a likelihood function. See Dietrich and List (2016).
} 
With the new form of conditionalisation, we can now show that the Geometric Conciliatory View commutes with conditionalisation. Take $\operatorname{Pr}_{P r_{1}, \ldots, P r_{n}}^{L}(\cdot)$ as the joint credence function obtained in the case where the individuals first conciliate with all others and subsequently update with a likelihood function $L . \operatorname{Pr}_{\operatorname{Pr}_{1}^{L}, \ldots, P r_{n}^{L}}(\cdot)$, on the other hand, stands for the joint credence function obtained in the case where the individuals first update with the likelihood function $L(\cdot)$ and then conciliate with the others. To show that the two functions yield the same outcome, it suffices to show that the two functions are proportional since any two probability functions that are proportional to one another must be identical. Consider the case in which the individuals make a conciliation first. Suppose there are $n$ individuals who disagree over their credence of a single possible world $\omega$. If they adopt the Geometric Conciliatory View and make a conciliation, the result would be $\operatorname{Pr}_{1}(\omega)^{w_{1}} \ldots \operatorname{Pr}_{n}(\omega)^{w_{n}}$. When they jointly receive a piece of evidence $E$ and derive a likelihood function $L(\cdot)$ from it, the outcome of updating would then be proportional to $\operatorname{Pr}_{1}(\omega)^{w_{1}} \cdots P r_{n}(\omega)^{w_{n}} L(\omega)$. On the other hand, when the individuals first update their credence functions with $L(\cdot)$, we have a set of updated credence functions $\operatorname{Pr}_{i}^{L}(\omega)$. Each function in the set is equivalent to $\operatorname{Pr}_{i}(\omega) L(\omega)$. The individuals later conciliate with the others and get the result $\left(\operatorname{Pr}_{1}(\omega) L(\omega)\right)^{w_{1}} \cdots\left(\operatorname{Pr}_{n}(\omega) L(\omega)\right)^{w_{n}}$, which is equivalent to $\operatorname{Pr}_{1}(\omega)^{w_{1}} \ldots \operatorname{Pr}_{n}(\omega)^{w_{n}} \cdot L(\omega)^{w_{1}+\cdots+w_{n}}$. Since $w_{1}, \ldots, w_{n}$ sum up to one, this result is proportional to $\operatorname{Pr}_{1}(\omega)^{w_{1}} \cdots \operatorname{Pr}_{n}(\omega)^{w_{n}} L(\omega)$, namely the result in the first case. We may hence conclude that the Geometric Conciliatory View does commute with conditionalisation. ${ }^{15}$

The problem of path dependence can be solved by adopting the Multiplicative Conciliatory View which suggests individuals to conciliate with multiplicative averaging: 16

\section{Definition 3 The Multiplicative Conciliatory View}

Given a case in which $n$ individuals $1, \ldots, n$ disagree over the proposition $P$, the Multiplicative EWV suggests the individuals involved to have credence

$$
\sum_{w \in P} c \cdot P r_{1}(\omega) \cdots P r_{n}(\omega)
$$

in the proposition $P$. The constant $c$ is a normalisation factor which guarantees that the sum of joint credences of all propositions equals to 1 .

$$
c=\frac{1}{\sum_{\omega^{\prime} \in \Omega} \operatorname{Pr}_{1}\left(\omega^{\prime}\right) \cdots P r_{n}\left(\omega^{\prime}\right)} .
$$

Two points should be noted: First, the Multiplicative Conciliatory View, like the Geometric Conciliatory View, also takes a single world as the input instead of a proposition.

\footnotetext{
15 This simplified proof is presented by Dietrich and List (2016). The original proof that geometric averaging commutes with conditionalisation is provided by Genest (1984).

16 Dietrich (2010) and Easwaran et al. (2016) both provide detailed analysis of the features of the multiplicative average function.
} 
Second, this view assigns equal weight to all the credence functions involved. Hence, we do not have to explicitly assign weight to each credence function.

It need not be proved that the Multiplicative Conciliatory View is path independent. Since multiplication is associative, it trivially holds that any conciliation made this way is also associative. ${ }^{17}$ The problem of path dependence, hence, would not occur for anyone conciliating with the Multiplicative Conciliatory View. ${ }^{18}$

Both the Geometric EWV and the Multiplicative EWV preserve one's judgement that two events are independent. The proof is also trivial. According to both the Geometric and the Multiplicative Conciliatory View, individuals should multiply their credences to make conciliation. Hence, the joint credence function of a group of peers assigns a value which is equivalent to the product of the credences of each individual. The problem of independence preservation can thus be solved. This point can be illustrated with a toy example: Consider a case involving two peers whose credences are represented respectively by the function $\operatorname{Pr}_{1}(\cdot)$ and $\operatorname{Pr}_{2}(\cdot)$. Let their joint credence function be $\operatorname{Pr}_{1+2}(\cdot)$. To show that both the Geometric and the Multiplicative Conciliatory View preserve their judgement that two propositions are independent, what we need to prove is that $\operatorname{Pr}_{1+2}(\cdot \wedge \cdot)$ is equivalent to $\operatorname{Pr}_{1+2}(\cdot) \operatorname{Pr}_{1+2}(\cdot)$ when the inputs are independent. According to the definition of the Geometric and the Multiplicative Conciliatory View, $\operatorname{Pr}_{1+2}(\cdot \wedge \cdot)$ is equivalent to $\operatorname{Pr}_{1}(\cdot \wedge \cdot) \operatorname{Pr}_{2}(\cdot \wedge \cdot)$. When the inputs are independent for both individuals, $\operatorname{Pr}_{1+2}(\cdot \wedge \cdot)$ is equivalent to $\operatorname{Pr}_{1}(\cdot) \operatorname{Pr}_{1}(\cdot) \operatorname{Pr}_{2}(\cdot) \operatorname{Pr}_{2}(\cdot)$. Since this formula is equivalent to $\operatorname{Pr}_{1+2}(\cdot) \operatorname{Pr}_{1+2}(\cdot)$, one's judgement that two propositions are independent can be well preserved.

In sum, the three formal deficiencies of the Linear EWV can be solved respectively by making conciliation with different nonlinear average functions. Since the proofs do not assume that all credence function involved are assigned with equal weight, the same formal result holds for every possible weight distribution. The Conciliatory View, hence, is free from the three formal deficiencies.

\section{Other features of nonlinear conciliation}

Although switching to nonlinear average functions may save the Conciliatory View from the three formal deficiencies, there is a standing worry that both nonlinear average functions introduced are far from ideal. Despite the Geometric Conciliatory View commutes with conditionalisation, it is still path dependent. The Multiplicative Conciliatory View, on the contrary, is path independent but not commutative with conditionalisation. Also, it should be noted that both nonlinear Conciliatory Views fail to be eventwise independent. That is, if we adopt the nonlinear Conciliatory Views, the

\footnotetext{
17 One may wonder whether the process of normalisation makes the Multiplicative Conciliatory View path dependent. To show that it does not, it suffices to show that the normalisation factors do not vary with the path. Consider a toy example in which $\operatorname{Pr}_{1}(\omega)=\alpha, \operatorname{Pr}_{2}(\omega)=\beta$ and $\operatorname{Pr}_{3}(\omega)=\gamma$. Given the Multiplicative Conciliatory View, one may obtain the result that $\operatorname{Pr}_{1+2}(\omega)=c \cdot \alpha \beta$ and $\operatorname{Pr}_{1+2+3}(\omega)=c^{\prime} \cdot c \cdot \alpha \beta \gamma$ where $c$ and $c^{\prime}$ stand respectively for the normalisation factor at each stage of conciliation. If we change the order of conciliation, we may derive the final result $\operatorname{Pr}_{1+3+2}(\omega)=c^{\prime \prime} \cdot c^{\prime \prime \prime} \cdot \alpha \beta \gamma$. By expanding the normalisation factors, one can see that $c \cdot c^{\prime}$ is equivalent to $c^{\prime \prime} \cdot c^{\prime \prime \prime}$. We may see that the process of normalisation does not make the Multiplicative Conciliatory View path dependent.
}

18 Easwaran et al. (2016, p. 16) provides a different proof to show the same result. 
collective credences of a group do not depend solely on the conciliating individuals' credences of the proposition but would be influenced by some other factors, such as the content of the agenda (Aczél and Wagner 1980; McConway 1981; Stewart and Quintana 2018; Dietrich and List 2016). ${ }^{19}$ Since no average function is perfect, some might still consider the Conciliatory View untenable.

This worry does not undermine the current approach but instead motivates us to embrace a pluralistic conception of conciliation. Since there does not exist a perfect average function which is applicable in every case, we should, in each specific case, adopt the average function that is most likely to avoid potential problems. For instance, if I am involved in a disagreement where I am sure that no further conciliation would take place but some new evidence may appear, I should adopt the Geometric Conciliatory View. By doing so, I can guarantee that the time I receive the evidence does not determine the outcome of conciliation. Similarly, if I know that someone owns the power of changing the agenda and I do not want the result of conciliation to be manipulated by the agenda setter, I should adopt the Linear Conciliatory View. The next step the conciliationists should take, therefore, is to create a taxonomy of disagreements. By attentively categorising various cases of disagreement, we may apply the right rule of conciliation when a disagreement occurs. The primary aim of this section, hence, is to demonstrate some features of the Geometric and Multiplicative EWV and specify the conditions under which they should be applied. ${ }^{20}$

To see other features of different average functions, we should first compare the outcomes of adopting different average functions in a simple scenario. Suppose one has 0.5 credence in a proposition and conciliates with a peer whose credence is $x .^{21}$ The outcomes of one's conciliation according to different average functions can be seen in Fig. 1. The value on the $x$ axis stands for one's peer's credence in the proposition under dispute, while the value on the $y$ axis stands for the outcome of conciliation. The solid and dotted lines respectively represent the result of adopting the Linear and Multiplicative EWV, while the S-curve represents the Geometric EWV.

19 A practical consequence of adopting the nonlinear Conciliatory Views is that the result of conciliation would be partly determined by the agenda, namely the set of propositions people disagree on. Since the nonlinear average functions are not eventwise independent, the joint credence a group has in a proposition may differ under different agendas. See McConway (1981) for the proof that linear averaging is the only function which satisfies the requirement of being eventwise independent. Adopting nonlinear Conciliatory Views, thus, makes the conciliating individuals vulnerable to manipulation by the agenda setters. An agenda setter may decide their joint credences in propositions by setting the agenda in a specific way. The reason is that given different agendas, the underlying set of worlds may change. Imagine a panel of climate scientists negotiating about a set of propositions on an agenda with the intention to decide their joint credences over the propositions. When someone expands the agenda with one more proposition, say whether there will be a hurricane next year, each world $\omega$ in the underlying set of possible worlds $\Omega$ would have to be replaced by two worlds: one which is a combination of $\omega$ and there being hurricanes next year, and another which combines $\omega$ and there being no hurricane next year. If one adopts the nonlinear Conciliatory Views, a change of agenda may lead to different outcomes of conciliation. Hence, the agenda setter may manipulate the result by setting the agenda in a specific way. This is another unacceptable result since, as indicated before, an ideal rule of conciliation should not leave space for manipulation.

${ }^{20}$ For the sake of simplicity, here I consider different EWVs, rather than different Conciliatory Views. The formal properties of different EWVs can be generalised to other forms of the Conciliatory View.

${ }^{21}$ It should be noted that one having a credence of a proposition is an abbreviation of one having a set of credences in the worlds where the proposition is true. 
Fig. 1 Individual with credence 0.5 compromises with a peer whose credence is $x$

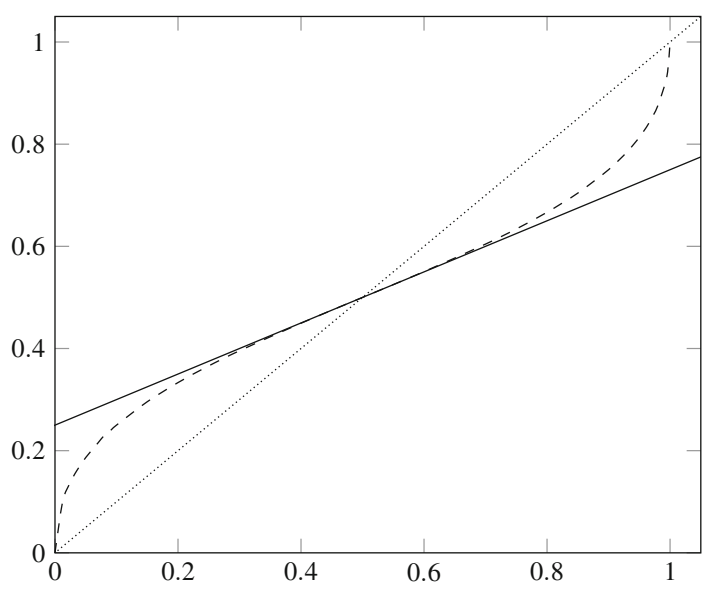

The Linear EWV, compared to the other two average functions, is the most resolute one since the disagreeing individuals who adopt this view never defer, in any sense, to each other. What they do is just split the difference between their credences.

The Multiplicative EWV generates a different result in this case. When one has 0.5 credence in a proposition and conciliates with their peer according to the Multiplicative EWV, the outcome would always equal to their peer's credence. That is, one always completely yields to their peer. ${ }^{22}$

The outcome of adopting the Geometric EWV is the most intriguing. It behaves like the Linear EWV when the peer's credence is moderate, but gradually deviates from the Linear EWV as the peer's credence gets close to the extreme. To be precise, when the peer's credence is below 0.1 or above 0.9 , the slope of the tangent line of the curve exceeds 1 . This feature implies that when one has moderate credence in a proposition and adopts the Geometric EWV, one would be inclined to yield to their peer when the peer is strongly opinionated on the issue. On the other hand, when they both have moderate credences, the outcome of conciliation stays moderate. Here, we have a rather interesting discovery: Based on the Geometric EWV, we can derive an objective threshold for credences that are high and low for an individual. In the case where one has 0.5 credence, the outcome of conciliation is closer to their peer's credence when their peer has credence above 0.9 or below 0.1 . One possible interpretation is that, given the Geometric EWV, a credence around 0.9 is objectively high for someone who has 0.5 credence in the same proposition. Similarly, a credence below 0.1 is objectively low for that individual. Epistemologists sometimes take 0.9 as the threshold for credences being significantly high. The Geometric EWV generates exactly this result.

Figure 2 presents a case in which one's credence in the proposition under dispute is 0.9 , while their peer's credence is again $x$. In this case, the Linear and the Geometric EWV behave in the same way, while the outcome of adopting the Multiplicative EWV is significantly different. When both the individual and their peer have 0.9 credence in the proposition, the outcome of conciliation, given the Multiplicative EWV, would

22 Easwaran et al. (2016) also mentioned this result. 
Fig. 2 Individual with credence 0.9 compromises with a peer whose credence is $x$

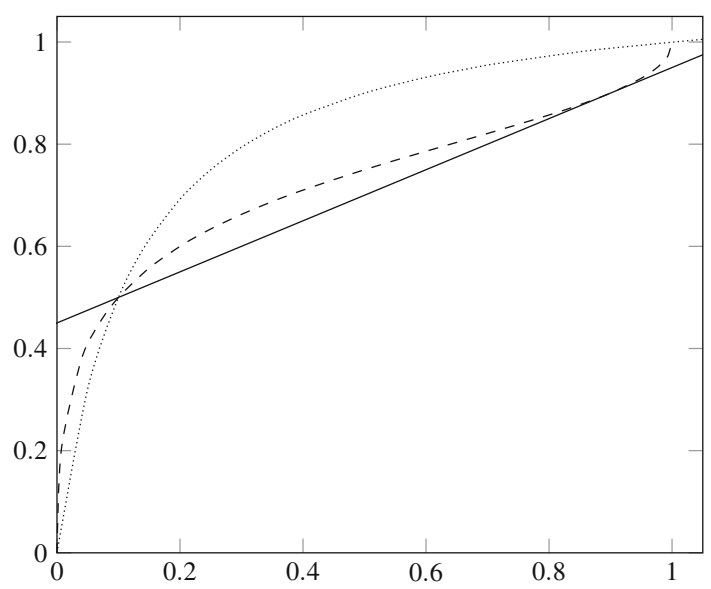

be greater than 0.9. This is a property (Easwaran et al. 2016) call synergy. When both the individuals' credences are high, the outcome would be even higher. Because of this property, the result generated by the Multiplicative EWV, compared to the other two EWVs, is always closer to the extreme.

What, then, is the correct way of making conciliation? Should one adopt the Geometric EWV and make a radical change of credence only when the peer is strongly opinionated? Or, should one adopt the Multiplicative EWV and sometimes completely surrender to the peer? As previously indicated, one should pick the EWV which is free from foreseeable problems. Moreover, a general guideline is to pick the rule according to how resolute one wants to be. As I point out, the Linear EWV, compared to the other two, is the most unwavering one. Individuals who adopt this view never completely surrender to their peers. The Multiplicative EWV, as we have seen, is the least resilient among the three, as it makes the individuals yield to their peers more frequently than any other views. Bearing this feature in mind, one may, in each specific scenario, choose the one that best suits the case.

One may think that in a standard case of peer disagreement, there is a perfect symmetry between the disagreeing individuals. Hence, the individuals involved should never yield to the others' opinion, which implies that the Linear EWV is the only acceptable option. In fact, even in these cases, one may choose to be less resilient about their credence. Here I want to highlight two factors that are crucial in deciding which EWV to adopt. One is the strength of the evidence one possesses which decides how resilient their credence is. The stronger their evidence is, the more unwilling one is to revise their credence. Another factor is the extent the evidence is shared. The more one knows about their peers' evidence before the conciliation, the more likely that one retains their original credence. ${ }^{23}$ This point can be illustrated by considering the case in which the individuals do not share their evidence. If one does not know whether their peer has evidence concerning a proposition, when one realises that their peer has some credence different from their own, one should be able to infer that their

\footnotetext{
23 As noted before, although deviate from the standard cases of disagreement in the literature, cases in which the individuals do not share all their evidence are still worth discussing. See Matheson (2014).
} 
peer does have some evidence. One may hence be inclined to defer to their peer. If one knows all the evidence their peer possesses, there is no reason to defer. With the two factors explained, we may see how different EWVs capture these intuitions.

Case 1 An individual has no evidence concerning a proposition $p$ and has 0.5 credence in it. ${ }^{24}$ They do not know if their peer has any evidence.

When the individual realises that their peer has different credence in the same proposition and hence disagrees with their, it is reasonable for them to think that their peer has better evidence and come up with more definite credence. In this case, one should completely defer to their peer. The Multiplicative EWV generates the correct result in this specific circumstance.

Case 2 An individual has some evidence concerning a proposition $p$ and has 0.5 credence in it. They know that their peer has some evidence, but do not know the strength of their peer's evidence.

When the individual realises that their peer's credence is not radically different from theirs, they could conciliate by moderately deferring to their peer. If one finds out that their peer's credence is very strong, they may realise that their peer's evidence must be rather conclusive. After all, they are equally good in evaluating the strength of the evidence they each possess. Hence, they should yield to their peer. Adopting the Geometric EWV generates the correct result.

One possible challenge to the solution is that their peer might come to have high credence with some weak evidence. If it is so, then it would be wrong for the individual to defer to their peer. However, by assuming the peerhood between them, this kind of cases should not occur. That is, a genuine epistemic peer would not come to have high credence based on insufficient evidence. True epistemic peers should be equally careful in evaluating the evidence available to them.

Apart from the two factors, there are some other important aspects that should be considered when choosing the proper rule for conciliation.

\subsection{Joint decision making}

The purpose of conciliation should be taken as a crucial factor in choosing the rule. On some occasions, the primary purpose of conciliation is to come up with a joint decision on whether to take a certain action. These cases are called action-disagreement. Different from belief-disagreement, a true resolution of an action-disagreement is an all or nothing thing, namely that the individuals involved either take action or not. There is no middle ground between the two options. Hence, an ideal rule of conciliation for an action-disagreement should be one which helps the individuals arriving at a consensus about whether to take action. Recall that the Multiplicative EWV has the feature of synergy, namely that one's credence enhances another if they point to the same direction. Because of this feature, when all the individuals' credences are above

\footnotetext{
24 It should be noted that I do not intend to imply that whenever one has no evidence concerning a proposition, one comes to have 0.5 credence over that proposition.
} 
0.5 , the outcome of conciliation with the Multiplicative EWV, compared to the other rules of conciliation, would be much closer to 1. Similarly, when all the individuals' credences are below 0.5, the outcome of adopting the Multiplicative EWV would be very close to 0 . We may, therefore, see that synergy makes it more likely for a group to form a consensus on whether to act. Compared to the unanimity preserving Linear EWV, the Multiplicative EWV better tackles cases of action-disagreement.

\subsection{Polarisation}

A phenomenon that worries many social epistemologists is belief polarisation. Consider a case where two individuals disagree about a controversial fact. When they are both exposed to some pieces of evidence concerning the disputed fact, it is natural for one to expect that the disagreement between them to be mitigated. However, empirical studies have shown that such expectation differs from what happens in reality. ${ }^{25}$ When the individuals are presented with evidence of a mixed character, they tend to strengthen their prior credence on the controversy. That is, one who believes that the disputed fact obtains would become even more certain about the fact, while the other one behaves in precisely the opposite way. Hence, sharing evidence may lead to an increase in the difference between their credences.

Polarisation gets even more severe when we escalate to the level of group disagreement. Suppose that two groups disagree over a proposition $p$. Members of group $A$ believe that $p$ is more likely to be true than not, while members of group $B$ believe the opposite. When the members of the two groups are exposed to some evidence concerning $p$, it can be expected that the two groups become more polarised than two individuals. First, what happens in the individual level would occur again: the members of $A$ come to have stronger credence in $p$, while members of $B$ revise their credences in the opposite way. Second, since the members are now grouped with others who share similar ideas, they would communicate with others and consolidate their credence over the disputed proposition. The two mechanisms make belief polarisation even more intense at the group level.

With the phenomenon of belief polarisation explained, we may now ask the question: Which rule should one adopt when the members of group $A$ intends to come up with a joint credence over $p$ ? The Multiplicative EWV is a bad option as its result is comparatively extreme. ${ }^{26}$ Suppose that group $A$ consists of four members. After being exposed to the evidence, half of the members have 0.7 credence in $p$ while another half have 0.8. Adopting the Multiplicative EWV, the outcome of their conciliation would be approximately 0.99 . Since this result is much higher than each of their prior credence, the difference between the joint credence of group $A$ and group $B$ becomes greater. Polarisation is further intensified with no substantial reason.

The Geometric EWV performs slightly better in this case. If members of group $A$ conciliate with the geometric EWV, the outcome would be approximately 0.78 , which

\footnotetext{
25 See Kelly (2008) and Sunstein (2017) for a full-fledged discussion about polarisation.

26 Here I assume that the members of a group do not come up with their credences independently. If they do, the Multiplicative EWV can be a good option for them to derive their joint credence as their credences can be amplified.
} 
is not very distant from their original credence. However, the Geometric EWV has the feature that when one of the members is strongly opinionated, others tend to defer to their credence. When one of the members have very high credence, the outcome would be dragged toward their credence. Hence, adopting the Geometric EWV may still heighten polarisation in certain situations.

The Linear EWV, compared to the nonlinear ones, is the most conservative rule of conciliation. In cases where people have a strong intention to avoid polarisation, the Linear EWV is the appropriate one to adopt. In sum, both the Geometric and the Multiplicative EWV run the risk of intensifying polarisation. Anyone involved in a disagreement which may lead to polarisation should be aware of the outcome of adopting a rule of conciliation.

Although there are still many other cases that could be discussed, the conclusion I would like to draw has been clearly illustrated by reviewing these possible cases of disagreement. There are cases where one should conditionally defer to their peers. Yet there are also cases where one should not defer in any sense. We may therefore conclude that there is no ultimately correct method of making conciliation. The decision concerning the average function one is supposed to apply must be based upon the specific situation one is involved.

\section{Conclusion}

The formal deficiencies of the Conciliatory View, as we have seen, stem from the misconception that there is only one way, namely linear averaging, that could be adopted to make conciliation. By selecting alternative average functions to make conciliation, the problems dissolve naturally.

Conciliating in a nonlinear way leads to some intriguing results. The most prominent one is that an individual may assign equal weight to all their epistemic peers yet makes a minimal revision of their own credence. If we interpret the weight one assigns to their peers as the extent one trusts the peers, we may derive the result that an individual can fully respect their peers' competence in a subject matter, but still retain their credence concerning the proposition in question. From this result, we may see that previous discussion concerning the Equal Weight View and the Conciliatory View are misguided. Before we argue whether we should conciliate, we should elaborate on the notion of conciliation we are using.

The study of different kinds of conciliation motivates us to embrace a pluralistic conception of conciliation. What we should do, hence, is to construct a taxonomy of disagreements carefully. By correctly sorting different cases, we may apply the right function to make conciliation for each case. The discussion over whether to conciliate makes sense only when we are talking about the best way of making conciliation.

Acknowledgements Thanks to Liam Kofi Bright, Margherita Harris, Christian List, Anna Mahtani, Sven Neth, Joe Roussos, two anonymous reviewers and the audiences at the Choice Group, FEW 2019, the 16th London-Berkeley Philosophy Graduate Conference and the 93th Joint Session of the Mind Association and the Aristotelian Society for helpful comments. 
Open Access This article is licensed under a Creative Commons Attribution 4.0 International License, which permits use, sharing, adaptation, distribution and reproduction in any medium or format, as long as you give appropriate credit to the original author(s) and the source, provide a link to the Creative Commons licence, and indicate if changes were made. The images or other third party material in this article are included in the article's Creative Commons licence, unless indicated otherwise in a credit line to the material. If material is not included in the article's Creative Commons licence and your intended use is not permitted by statutory regulation or exceeds the permitted use, you will need to obtain permission directly from the copyright holder. To view a copy of this licence, visit http://creativecommons.org/licenses/by/4.0/.

\section{References}

Aczél, J., \& Wagner, C. (1980). A characterization of weighted arithmetic means. SIAM Journal on Matrix Analysis Applications, 1, 259-260.

Arntzenius, F. (2003). Some problems for conditionalization and reflection. Journal of Philosophy, 100(7), 356-370. https://doi.org/10.5840/jphil2003100729.

Bacchus, F., Kyburg, H. E, Jr., \& Thalos, M. (1990). Against conditionalization. Synthese, 85(3), 475-506.

Bradley, R., Dietrich, F., \& List, C. (2014). Aggregating causal judgments. Philosophy of Science, 81(4), 491-515.

Briggs, R., \& Pettigrew, R. (2020). An accuracy-dominance argument for conditionalization. Noûs, 54(1), 162-181. https://doi.org/10.1111/nous.12258.

Christensen, D. (2007). Epistemology of disagreement: The good news. Philosophical Review, 116(2), $187-217$.

Dietrich, F. (2010). Bayesian group belief. Social Choice and Welfare, 35(4), 595-626.

Dietrich, F., \& List, C. (2016). Probabilistic opinion pooling. In A. Hajek \& C. Hitchcock (Eds.), Oxford handbook of philosophy and probability. Oxford: Oxford University Press.

Easwaran, K., Fenton-Glynn, L., Hitchcock, C., \& Velasco, J. D. (2016). Updating on the credences of others: Disagreement, agreement, and synergy. Philosophers' Imprint, 16, 1-39.

Elga, A. (2007). Reflection and disagreement. Noûs, 41(3), 478-502. https://doi.org/10.1111/j.1468-0068. 2007.00656.x.

Elkin, L., \& Wheeler, G. (2018). Resolving peer disagreements through imprecise probabilities. Noûs, 52(2), 260-278. https://doi.org/10.1111/nous.12143.

Feldman, R. (2006). Epistemological puzzles about disagreement. In S. Hetherington (Ed.), Epistemology futures (pp. 216-236). Oxford: Oxford University Press.

Fitelson, B., \& Jehle, D. (2009). What is the 'equal weight view'? Episteme, 6(3), 280-293.

Gardiner, G. (2014). The commutativity of evidence: A problem for conciliatory views of peer disagreement. Episteme, 11(1), 83-95.

Genest, C. (1984). A characterization theorem for externally Bayesian groups. The Annals of Statistics, 12(3), 1100-1105. https://doi.org/10.1214/aos/1176346726.

Greaves, H., \& Wallace, D. (2005). Justifying conditionalization: Conditionalization maximizes expected epistemic utility. Mind, 115(459), 607-632.

Hild, M. (1998). The coherence argument against conditionalization. Synthese, 115(2), 229-258. https:// doi.org/10.1023/A:1005082908147.

Kelly, T. (2008). Disagreement, dogmatism, and belief polarization. Journal of Philosophy, 105(10), 611633. https://doi.org/10.5840/jphil20081051024.

Kelly, T. (2010). Peer disagreement and higher order evidence. In A. I. Goldman \& D. Whitcomb (Eds.), Social epistemology: Essential readings (pp. 183-217). Oxford: Oxford University Press.

King, N. L. (2012). Disagreement: What's the problem? Or a good peer is hard to find. Philosophy and Phenomenological Research, 85(2), 249-272.

Martini, C., Sprenger, J., \& Colyvan, M. (2013). Resolving disagreement through mutual respect. Erkenntnis, 78(4), 881-898.

Matheson, J. (2014). Disagreement: Idealized and everyday. In J. M. R. Vitz (Ed.), The Ethics of Belief: Individual and Social (pp. 315-330). Oxford: Oxford University Press.

McConway, K. J. (1981). Marginalization and linear opinion pools. Journal of the American Statistical Association, 76(374), 410-414. https://doi.org/10.1080/01621459.1981.10477661. 
Pettigrew, R. (2019). What is conditionalization, and why should we do it? Philosophical Studies, 1-37. https://doi.org/10.1007/s11098-019-01377-y.

Pettit, P. (2006). When to defer to majority testimony-And when not. Analysis, 66(3), 179-187.

Stewart, R. T., \& Quintana, I. O. (2018). Probabilistic opinion pooling with imprecise probabilities. Journal of Philosophical Logic, 47(1), 17-45. https://doi.org/10.1007/s10992-016-9415-9.

Sunstein, C. R. (2017). Polarization. In \#republic: Divided democracy in the age of social media (Chap. 3 , pp. 59-97). Princeton University Press.

van Inwagen, P. (1996). It is wrong, everywhere, always, for anyone, to believe anything upon insufficient evidence. In J. Jordan \& D. Howard-Snyder (Eds.), Faith, freedom and rationality, savage (pp. 137154). Lanham, MD: Rowman and Littlefield.

Wilson, A. (2010). Disagreement, equal weight and commutativity. Philosophical Studies, 149(3), 321-326.

Publisher's Note Springer Nature remains neutral with regard to jurisdictional claims in published maps and institutional affiliations. 